\title{
The Role of Grain Size in He Bubble Formation: Implications for Swelling Resistance
}

O. El-Atwani ${ }^{1}$, J. Nathaniel $\mathrm{II}^{1}$, A.C. Leff ${ }^{1}$, B.R. Muntifering ${ }^{2}$, J . K. Baldwin ${ }^{3}$, K. Hattar ${ }^{2}$, M.L. Taheri $^{1}$

1. Drexel University, Department of Materials Science \& Engineering, Philadelphia, PA, USA.

2. Department of Radiation Solid Interactions, Sandia National Laboratories, NM, USA.

${ }^{3 .}$ Center for Integrated Nanotechnologies, Los Alamos National Laboratory, Los Alamos, NM, USA

\begin{abstract}
Nanocrystalline metals are postulated as radiation resistant materials due to their high defect and particle (e.g. Helium) sink density. Here, the performance of nanocrystalline iron films is investigated in-situ in a transmission electron microscope (TEM) using He irradiation at $700 \mathrm{~K}$. Automated crystal orientation mapping is used in concert with in-situ TEM to explore the role of grain orientation and grain boundary character on bubble density trends. Bubble density as a function of three key grain size regimes is demonstrated. While the overall trend revealed an increase in bubble density up to a saturation value, grains with areas ranging from 3000-7500 $\mathrm{nm}^{2}$ show a scattered distribution. An extrapolated swelling resistance based on bubble size and areal density indicated that grains with sizes less than $2000 \mathrm{~nm}^{2}$ possess the greatest apparent resistance. Moreover, denuded zones are found to be independent of grain size, grain orientation, and grain boundary misorientation angle.
\end{abstract}

Keywords: bubble formation, nanocrystalline, denuded zone, swelling 


\section{Introduction}

Material degradation at high radiation doses [1] and the extreme irradiation environments such as those that occur in fission and fusion energy systems occurs via the nucleation of defects. These include bubbles, voids, and dislocation loops that alter the properties of the irradiated materials [2-7] and ultimately decrease performance [3]. Such degradation occurs in both fusion and fission materials. For example, in tungsten, of interest in fusion, damage from He ions can produce morphological changes [8-10] that cause enhanced erosion that can eventually degrade the plasma performance through radiation cooling [11]. Similarly, fission materials exposed to neutron irradiation and fission fragments can degrade through swelling, embrittlement, and hardening $[3,5]$. Nanocrystalline materials with their improved mechanical properties are more tolerant to high radiation doses [12-14]. Their increased grain boundary population translates to an increased defect sink density that could enhance irradiation induced defect annihilation [15$18]$.

Grain boundaries, acting as defect sinks [19-21], may assist in suppressing or controlling radiation damage [22]. Consequently, it has been speculated that a high grain boundary density could increase the irradiation dose threshold necessary to accumulate a high enough density of defects to significantly change the properties of the material [23]. Several experimental and theoretical studies have investigated the performance of ultra-fine grained and nanocrystalline materials with respect to dislocation loop, bubble, and void densities [16-18, 24-40], as well as morphological instabilities such as nanostructure formation $[10,23,41]$. In several metals (Copper [29], Nickel [29] and Gold [30]), nanocrystalline microstructures have exhibited higher radiation tolerance than coarse grained microstructures as measured by irradiation induced cluster defect density. Similarly, regarding bubble formation and evolution in BCC 
nanocrystalline materials, it has been demonstrated experimentally [17] that nanocrystalline tungsten grains lead to lower helium bubble density in the grain matrix when compared to ultrafine grains. This observation is in agreement with simulations performed by Sefta et al. [39] which demonstrated higher helium retention in bicrystalline tungsten materials compared to single crystalline tungsten. Molecular dynamics simulations performed by [16, 19, 24-26, 42] demonstrated the role of grain boundaries in annihilating interstitial defects and vacancies. Bai $e t$ al.[25] demonstrated an enhanced defect annihilation process through rapid interstitial defect absorption and reemission (and subsequent combination) with vacancies approaching grain boundaries. A large scatter of defect densities as a function of grain size has been observed, however, which has been attributed to the dependence of boundary sink efficiency on grain boundary character [27] and other interfacial deviations [43].

Experimental evidence of the dependence of bubble formation and swelling due to helium implantation on grain size is crucial in investigating the performance of candidate nanocrystalline materials for future nuclear applications and the development of mesoscale predictive models. When displacement energies are sufficient to produce vacancies that bind to helium in stable helium-vacancy complexes, this occurs in timescales as short as hundreds of picoseconds [44]. For example, in pure iron at intermediate temperatures $(>573 \mathrm{~K})$ small vacancy clusters formed by migration of individual vacancies are unstable [45] and need helium to stabilize them. The growth of these helium-vacancy clusters lead to bubble formation that degrades mechanical properties [46, 47]. Interstitial helium atoms, due to their low migration energy $(0.06 \mathrm{eV})$ [48], are predicted to agglomerate quickly and eject interstitial $\mathrm{Fe}$ atoms, eventually leading to the formation of helium-vacancy clusters, and eventually bubbles. Small helium-vacancy clusters are also mobile at temperatures over $573 \mathrm{~K}$ [45] and therefore, their 
coalescence can enhance bubble formation. The relationship between bubble formation and grain size are relatively unknown, especially in nanocrystalline size regimes.

In this work, nanocrystalline $\mathrm{Fe}$ is used as a model $\mathrm{BCC}$ material to investigate the performance of high sink density materials under helium irradiation in-situ in the transmission electron microscope (TEM), coupled with automated crystal orientation mapping (ACOM) [49] for grain orientation analysis. A comparison of bubble densities in nanocrystalline to ultra-fine grained size regimes shows that bubble density increases with grain size in the nanocrystalline regime, but scatters in the transition from the nanocrystalline to the ultrafine grained regime; this appears to reach a saturation value in the ultra-fine grained regime. An apparent swelling, as extrapolated from the bubble density and size data, is shown to follow similar grain size trends, as expected. These results suggest that nanocrystalline materials have potential for use in extreme environments where swelling due to void or bubble formation is a concern.

\section{Experimental}

\section{Sample Preparation}

Nanocrystalline Fe films (approximately $100 \mathrm{~nm}$ nominal thickness) [50, 51] were sputter-deposited on $\mathrm{NaCl}$ substrates. The details of the film preparation conditions are described elsewhere by Vetterick et al. [50]. The films were then floated in water onto 300 mesh molybdenum TEM grids (Tedpella, Inc.) and annealed to $873 \mathrm{~K}$ in-situ in a JEOL $2100 \mathrm{LaB}_{6}$ TEM using a Gatan 628 single-tilt heating stage to reach an equilibrium microstructure. The overall microstructure consists of both nanocrystalline ( $<100 \mathrm{~nm}$ diameter) and ultra-fine (100$500 \mathrm{~nm}$ diameter) grains. The supplemental material briefly describes the calculation of the average grain size. 


\section{Irradiation and Microstructural Characterization}

In-situ TEM irradiation with He was performed using the In-situ Ion Irradiation TEM facility [52] at Sandia National Laboratories with He ions at $700 \mathrm{~K}$ and a dose rate of $8.74 \times 10^{17}$ $\mathrm{He} / \mathrm{m}^{2}-\mathrm{s}^{-}$to $2.8 \times 10^{21} \mathrm{He} / \mathrm{m}^{2}$. The Monte Carlo computer code Stopping Range of Ions in Matter (SRIM) (version 2013) [53] was used to perform analysis to determine the distribution of damage introduced into the materials (Figure 1). The mean ion range in the depth direction is approximately $35 \mathrm{~nm}$ for $10 \mathrm{keV} \mathrm{He}{ }^{+}$irradiation at 60 degrees incidence, which implies that the He ions will remain implanted in the sample rather than being transmitted through it. Assuming $40 \mathrm{eV}$ as a displacement damage energy threshold [54] and a $100 \mathrm{~nm}$-film thickness, the detailed calculation with full damage cascades option in SRIM demonstrated an average vacancy production of approximately 31 vacancies/ion. The final helium concentration, using a maximum implanted depth of $80 \mathrm{~nm}$ as shown in Figure 1 is then found to be $3.5 \times 10^{28} \mathrm{~m}^{-3}$ and the $\mathrm{He} / \mathrm{Fe}$ atoms ratio is approximately 0.41 (in the implanted region). At peak depth (35 nm) as shown in Figure 1 the vacancy production was predicted to be 0.38 vacancies/ion/nm and the corresponding damage value was approximately 12.5 dpa.

He bubble density, location, and morphology were characterized using bright-field TEM imaging in a JEOL $2100 \mathrm{LaB}_{6}$ TEM at under-focused (bubbles appears bright) and over-focused (bubbles appear dark) conditions and Fresnel imaging conditions plus additional ACOM analysis [55]. ACOM was performed with the NanoMEGAS ASTAR precession diffraction system [49], using a $10 \mathrm{~nm}$ spot size and a $2 \mathrm{~nm}$ step size. 
A total of 32 grains from three separate regions on the film were quantified using underfocused and over-focused bright-field images. For orientation analysis, index values ranged from 172 to 1100 and grains that indexed with a reliability of less than 20 were ignored. An example of an inverse pole-figure (IPF) map with the corresponding index and reliability maps produced using the NanoMEGAS ASTAR system is given in the supplemental section.

To determine bubble areal density, several $15 \mathrm{~nm}$ diameter circles were drawn randomly on each grain using ImageJ software [56]. The number of bubbles in each circle were counted and the density distribution was taken. Bubble areas were estimated by drawing a circle with ImageJ software around the perimeter of bubbles chosen randomly on every grain and an average area taken. To eliminate errors in grain size calculations due to differences in grain morphologies, the bubble density and bubble diameter graphs are plotted as a function of projected grain area. It should be mentioned that the projected He depth peak (Figure 1), is much less than the nominal thickness of the film (100) $\mathrm{nm}$. This is an important consideration when comparing phenomena (such as bubble formation) that can be affected by the proximity to free surfaces in the foil. To minimize the effect of film thickness, the grains used for bubble density and size quantifications were chosen from close regions in the film where mass thickness contrast was not observed in the TEM

Details about calculations of bubble density, bubble area, and extrapolated swelling due to cavity formation are outlined in the supplemental section. 


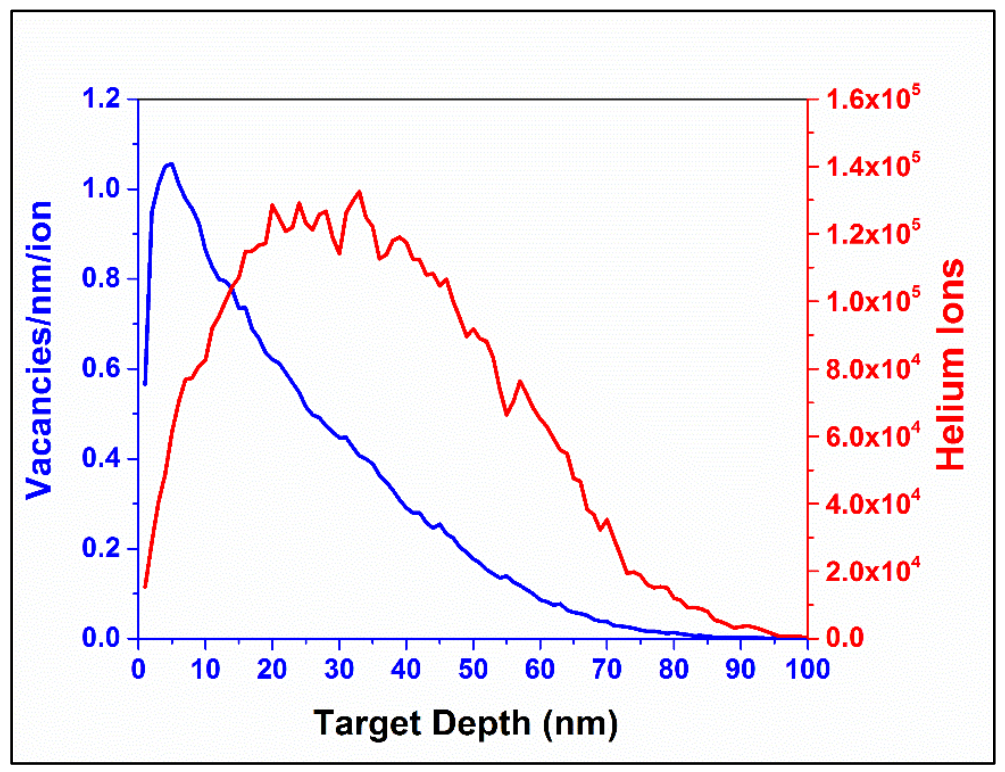

Figure 1: SRIM calculations performed for $10 \mathrm{keV}$ helium ions bombarding $100 \mathrm{~nm}$ iron sample at $60^{\circ}$ incidence to the ion beam showing predicted: Ion distriubtion (red curve) and vacancy production (blue curve) vs target depth.

\section{Results and Discussion}

\section{Bubble Density vs Grain Size}

After irradiation, both grain boundaries and grain interiors contained small cavities assumed to be He bubbles based on previous coarse-grain Fe characterization [57, 58]. Figure 2 is a low magnification, under-focused, bright-field TEM image, with a corresponding IPF map, that shows some of the grains and areas analyzed to determine bubble densities. 

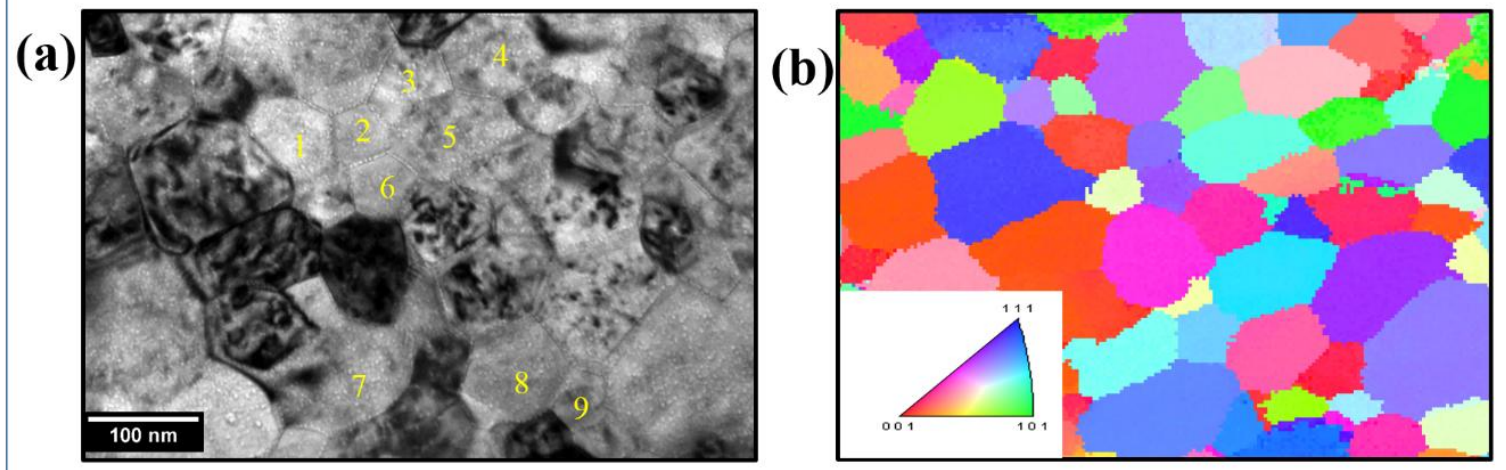

Figure 2: (a): Underfocused (bubbles are with white contrast) TEM bright field image of regions in the sample in which grains were analyzed. (b): a corresponding [001] IPF map from the same region shown in (a), demonstrating an equiaxed structure with random texture.

The grain size, morphology, and texture observed in this region is representative of the specimen as a whole. TEM micrographs of all grains (32 grains total) quantified in this study are displayed in the supplemental section. The plot in Figure 3a shows the logarithmic increase in bubble density with grain area. Three distinct regimes were identified for the following ranges of grain size: (1) below $3000 \mathrm{~nm}^{2}$, (2) between 3000 and $7500 \mathrm{~nm}^{2}$ and (3) over $7500 \mathrm{~nm}^{2}$. In the first category $\left(<3000 \mathrm{~nm}^{2}\right)$, an increase in bubble density as a function of grain size is clear. However, the results in the second regime are scattered, which could be attributed to different grain and grain boundary behavior in absorbing defects and trapping helium particles. In the third regime $\left(>7500 \mathrm{~nm}^{2}\right)$, the density saturation maximum and only a slight increase in density is observed as grain size increases.

In regime $1\left(<3000 \mathrm{~nm}^{2}\right)$, while the density is shown to increase with grain size, grains of less than $400 \mathrm{~nm}^{2}$ (or $20 \mathrm{~nm}$ diameter) do not contain bubbles in their matrices (only at grain boundaries), as shown in Figure $3 \mathrm{c}$ and $\mathrm{d}$. This could be caused by a decreased probability of helium-vacancy complex formation (which eventually leads to bubble nucleation and growth) 
due to higher sink strength of the overall microstructure as discussed previously [17]. Small Hevacancy complexes, $\mathrm{He}_{\mathrm{n}} \mathrm{V}_{\mathrm{m}}$ with low $\mathrm{n} / \mathrm{m}$ ratios can be unstable $[58,59]$ and require a critical number of helium atoms to stabilize the complex and form bubbles, which can be diminished at small grain sizes due to helium trapping on the boundary[60].

In contrast, the bubble densities in regime 2 (between 3000 and $7500 \mathrm{~nm}^{2}$ ) were scattered and the edge of regime 2 and regime 3 represents the threshold of nanocrystalline grain size at which bubble density starts to decrease. A study by El-Atwani et al.[17] on another BCC nanocrystalline material (a nanocrystalline ultrafine grained tungsten) showed a grain size threshold $(60 \mathrm{~nm})$ of lower bubble density in the matrix that agreed with this study. However, the microstructure of the tungsten in that case, which was prepared through severe plastic deformation, differs greatly from the columnar microstructure of the Fe thin film sample in this study.

In regime 3 (over $7500 \mathrm{~nm}^{2}$ ), the value of bubble density approaches a saturation value suggesting a balance between helium-vacancy complex formation and absorption of vacancies/complexes by grain boundaries.

Figure $3 b$ shows bubble diameter vs grain area for the same grains quantified in Figure 3a. No trend was observed as a function of grain size and the average bubble diameter was approximately $3 \mathrm{~nm}$ with a standard deviation of $0.24 \mathrm{~nm}$ and standard error of the mean of 0.042 . 

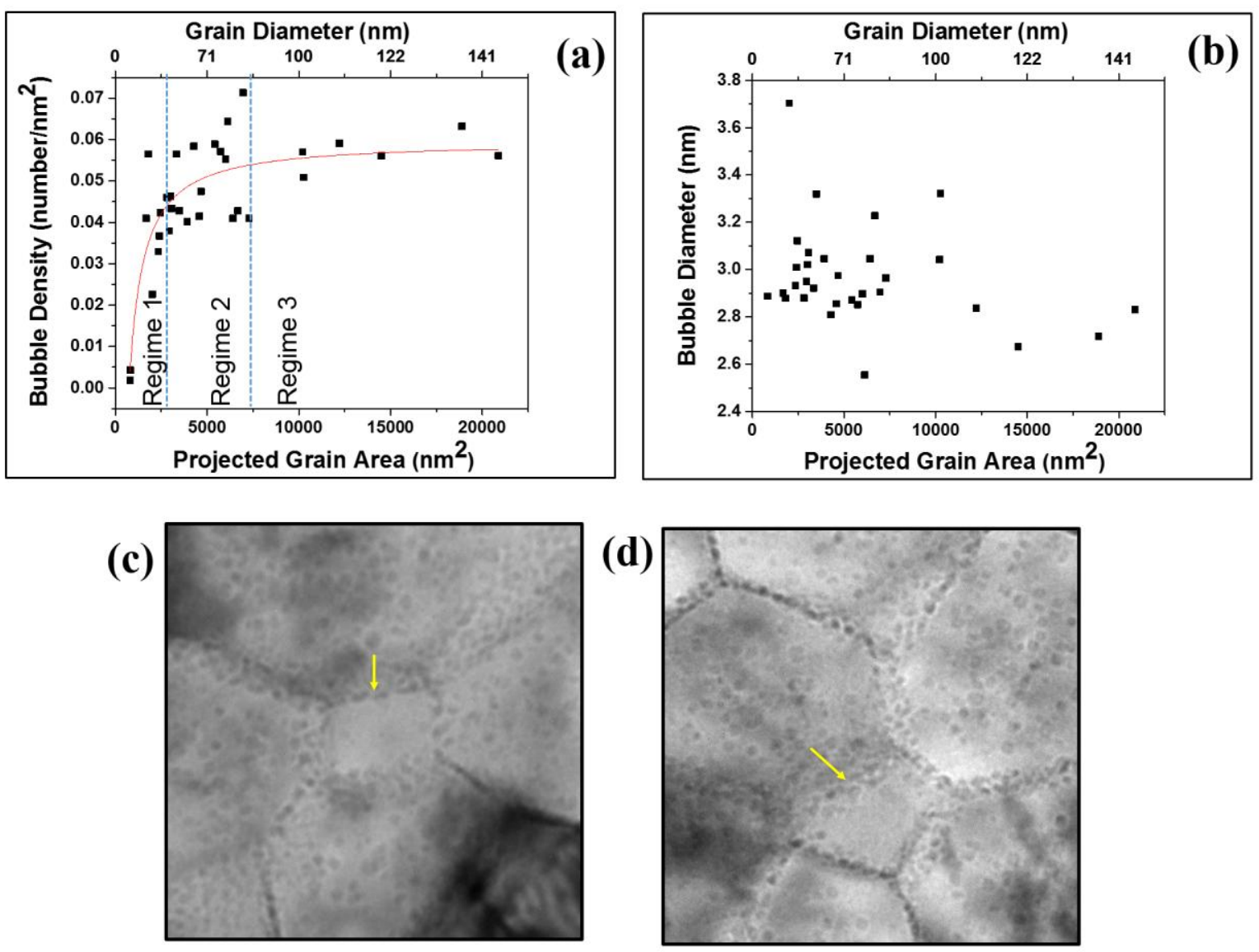

\section{$20 \mathrm{~nm}$}

Figure 3: (a): Bubble density (number/ $/ \mathrm{nm}^{2}$ ) vs grain size (projected grain area) with yellow fitting showing the trend and three grain size regimes. (b) Average bubble diameter vs grain size (projected grain area). (c) and (d) Bright field overfocues (bubbles are with dark contrast) TEM images showing two grains (pointed by yellow arrows) of less than $20 \mathrm{~nm}$ with no bubbles in the grain matrix. Upper $x$-axis in (a) and (b) represents the grain diameter (square root of the grain area).

\section{Role of Grain Orientation and Grain Boundary Type}

The scattering behavior observed in this second grain size regime could be a result of a variety of effects including, but not limited to, channeling effects due to grain orientation, grain boundary character, or local strain at grain boundaries. All of these effects could play a role in defect absorption at these interfaces. Channeling of helium atoms may lead to varying helium 
implantation in different grains and ultimately varying defect quantities [61, 62]. To study possible defect density variation due to grain orientation, the bubble density vs grain size including grain orientation is plotted (Figure 4a). The same figure for 001 grain orientation is also shown (Figure 4b). Using the Lindhard-Onderdilendan (L-O) theory [63, 64], and the description provided by Kempshall et al., on Ga ion channeling during focused ion beam (FIB) milling [65], the non-channeled factor, for the same ion penetrating the same material element, for different directions is proportional to $\mathrm{d}^{3 / 2}$ where $\mathrm{d}$ is the distance between the atoms in the index direction. Therefore, one expects the non-channeled ratio of [001] direction to be 0.59 and 1.24 of the [110] and [111] directions respectively if the ions are directed along these directions. We attempted to study channeling effects by determining the grain orientations relative to the [001] specimen axis. (Figure 4). The irradiation and the post-irradiation characterization were performed at different types and using different microscopes. Therefore, deviations can occur since irradiation was performed at 60 degrees incidence while ACOM was performed using a beam rotating around a spot normal to the sample. Grains of [001] orientation (Figure 4b), however, should not be affected by such deviations. The second grain size category in Figure 4 shows that grains of similar grain size and orientation can have different bubble densities. This excludes channeling effects on the trend in bubble density and the scattering behavior in regime 2. 

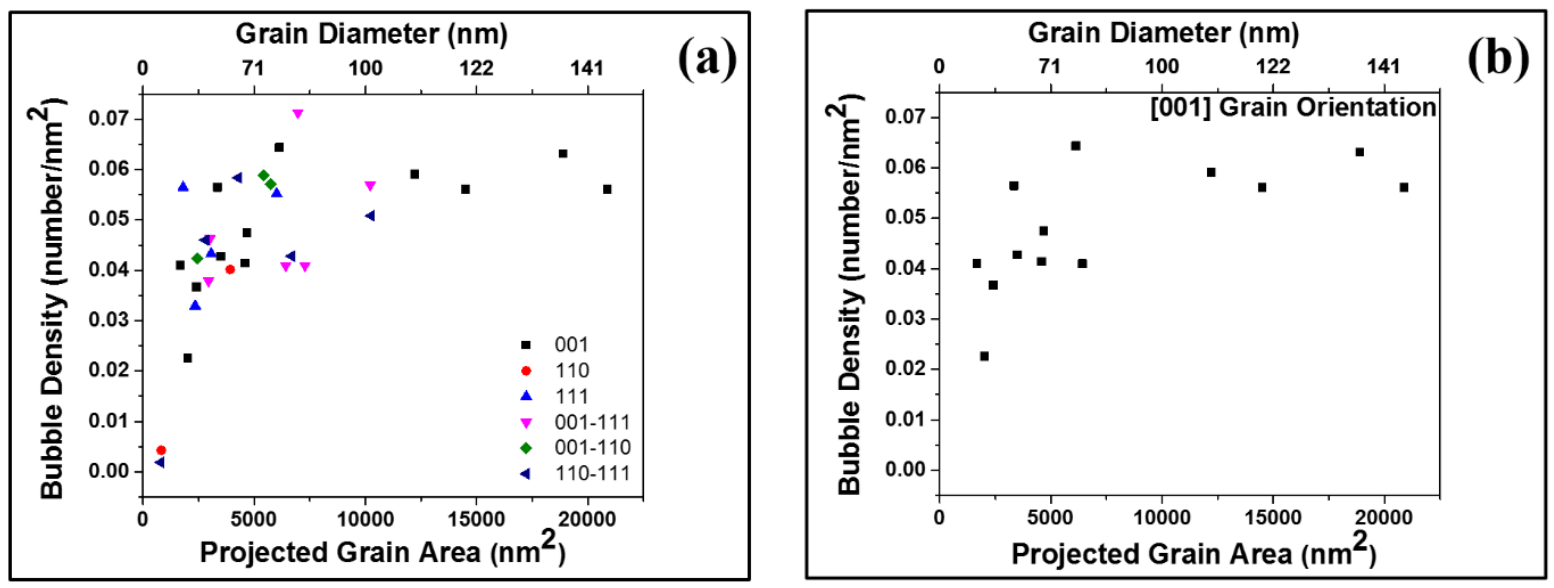

Figure 4: (a) Bubble density (number $/ \mathrm{nm}^{2}$ ) vs grain size (projected grain area) extracted form ACOM data for grains of 6 differing orientations, color shows orientation with respect to speciman normal. (b) ) Bubble density $\left(\mathrm{no} / \mathrm{nm}^{3}\right)$ vs grain size for [001] grain orientation. Upper $\mathrm{x}$-axis represents the grain diameter (square root of the grain area).

Several works have shown the importance of grain boundary plane, in determining sink efficiency and point defect interaction [19, 26, 42, 66, 67]. Grain boundary sink efficiency is thought to depend on grain boundary crystallography (overall character) $[26,66]$, and the sinkdefect absorption efficiency can lead to denuded zone formation [26, 68].

The results here confirm, however, that the misorientation angle is an insufficient descriptor for examining the relationship between grain boundary character and sink efficiency. In this work, bubble denuded zones were observed at both low and high angle grain boundaries. No correlation between misorientation angle and denuded zone formation was observed, as can be seen in the TEM micrographs shown in Figure 5. The scatter could be attributed to the fact that the misorientation angle only determines three out of five of the macroscopic degrees of freedom of grain boundary character, with the other two determined by the grain boundary plane. 

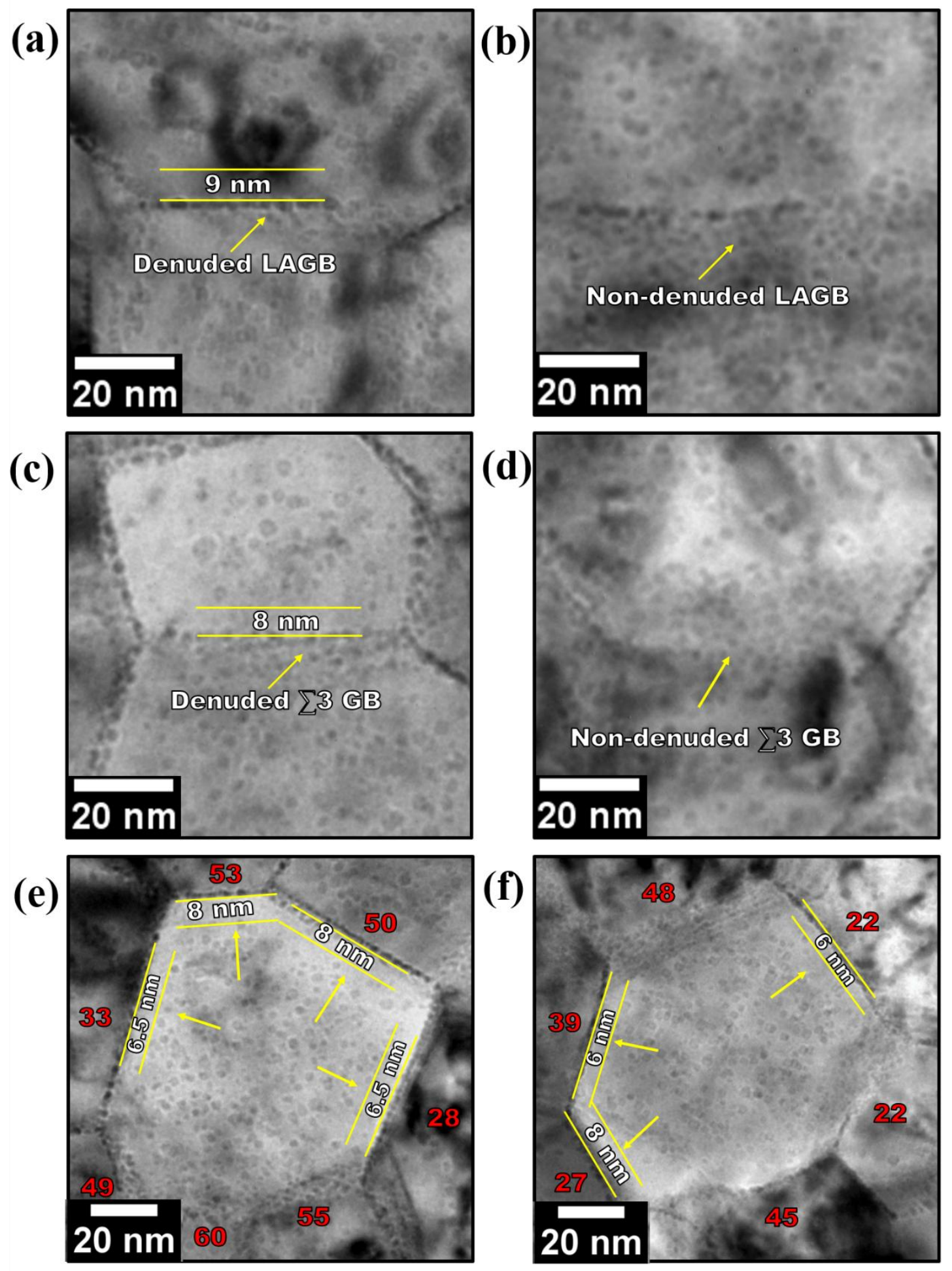

Figure 5: Overfocused (bubbles are with dark contrast) bright field TEM images showing: (a) and (b) denuded and non-denuded low angle grain boundaries (LAGB) pointed by yellow arrows respectively. (c) and (d) denuded and non-denuded sigma ( $\left.\sum 3\right)$ boundaries pointed by yellow 
arrows respectively (e) and (f) denuded and non-denuded grain boundaries for different misorientation angles (shown in red), and the denuded boundaries were pointed with yellow arrows.

\section{Comparison of Nanocrystalline Grains with Fine Grains and Implications for Swelling}

A fundamental question regarding radiation tolerance of nanocrystalline materials in the presence of He irradiation is whether or not a high grain boundary density leads to a lower bubble density due to boundaries acting as defect and particle sinks. The low migration energy of interstitial helium atoms allows them to move both quickly and freely to trapping sites. Grain boundaries are considered 2-D He trapping sites which offer limited He diffusion compared to the matrix (3D trapping) [60] and could be considered bubble trapping sites [69, 70].

A direct comparison of fine grained and nanocrystalline grains in the same specimen helps to provide insight into the dependence of bubble density on grain boundary density. Figure 8 shows a region of the sample that experienced grain growth during irradiation. Although the sample was annealed at $873 \mathrm{~K}$ prior to irradiation at $700 \mathrm{~K}$ continued grain growth and the formation of a bimodal grain size distribution is possible due to irradiation enhanced abnormal grain growth $[71,72]$. This process occurs via irradiation enhanced diffusion [73], which leads to an increased defect and grain boundary mobility $[74,75]$ as well as higher density of dislocation loops due to a trap-mutation process from the growing bubbles [76]. Abnormal grain growth has previously been observed in metallic samples irradiated with helium [23]. In our experiment, the observed abnormal grain growth occurred during the early stage of irradiation. As such, the region of the specimen containing a bimodal distribution of grain sizes can be used as a test case for a grain size dependence of bubble density. The diameter of the large grains was greater than $500 \mathrm{~nm}$. 
Bubble density and size measurements were performed on the large grains (grain size $>$ $500 \mathrm{~nm}$ ) to examine the effect of small grain size on bubble density and swelling. Careful bubble size imaging (Fresnel imaging) and analysis is critical for this comparison, and such imaging in the microscope was performed to minimize effects of imaging conditions on bubble size determination. The bubble density for the fine grains was found to be $0.112 / \mathrm{nm}^{2}$ which is 22 times larger than the lowest bubble density and 1.7 times larger than the saturation bubble density (Figure 3a) in the nanocrystalline regime.

The average bubble diameter in the fine grains was $2.1 \mathrm{~nm}$ (with a standard deviation of $0.25 \mathrm{~nm}$ and standard error of the mean of 0.035 ) compared to $3 \mathrm{~nm}$ in the nanocrystalline regime. This can be understood by considering the nucleation and growth of bubbles. During nucleation helium binds to a vacancy to form a helium-vacancy complex. In the nanocrystalline regime, the fast migration of helium atoms to the boundaries, due to short diffusion length, results in fewer nucleation sites. Therefore, during grain growth, the probability of a helium atom attaching to an existing helium-vacancy complex increases in the nanocrystalline regime. And at high sink densities (e.g., nanocrystalline samples), less recombination of interstitials with vacancies occurs [77] and therefore bubbles could get larger.

Simple void growth kinetics in irradiated materials $[78,79]$ provides a better explanation. Void growth was described using point defect balance equations and void growth kinetics by the following equation [79]:

$$
\frac{d r_{c}}{d t}=\frac{\Omega}{r_{c}}\left[Z_{v}^{c} D_{v} C_{v}-Z_{i}^{c} D_{i} C_{i}-Z_{v}^{c} D_{v} C_{v}^{e}\left(r_{c}\right)\right]
$$

Where $r_{c}$ is the cavity radius, $\Omega$ is the atomic volume, $D$ is the diffusion coefficients of point defects, $Z^{c}$ is the cavity capturing efficiency of point defects, $C$ is the concentrations of point 
defects and $C^{e}\left(r_{c}\right)$ is the thermal concentration of point defects at $r_{c}$, i denotes interstitials and $\mathrm{v}$ denotes vacancies. The first and second term in the right-hand side of equation (1) describes the vacancy and interstitial flux to the cavity, respectively. The third term describes the thermal emission of the vacancies from the cavity.

One possible hypothesis is that interstitial fast and biased [24] annihilation to the grain boundaries in the nanocrystalline regime would decrease the second term in the equation (interstitial flux to the cavity). Then the void radius will subsequently increase.
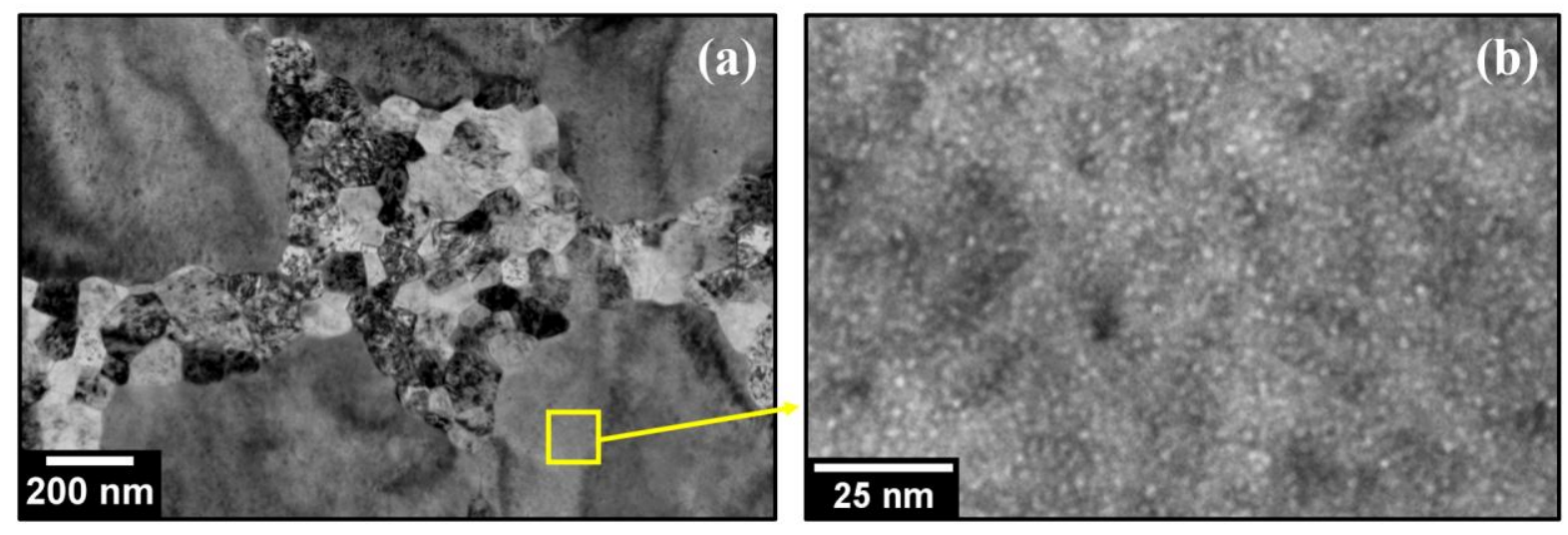

Figure 6: (a) TEM image of recrystallized regions in the sample showing bimodal grain distribution (b) Underfocused (bubbles are with white contrast) bright field TEM image of the bubbles in the matrix of the fine grains.

The lower bubble density in the nanocrystalline regime may not ensure less radiation damage, since bubble or cavity sizes in the nanocrystalline can differ from those in the larger grains as discussed above. Cavity formation can lead to volumetric swelling. Recent work by Muntifering et al.[43] suggested only a limited reduction of swelling in pulsed laser deposited nanocrystalline Nickel. 
In this work, swelling was not measured directly. Instead, we attempt to study the implication of bubble formation on swelling and the possible advantages of nanocrystalline grains by estimating the overall volume of bubbles formed in the sample during irradiation. We acknowledge that our swelling calculations, illustrated below, can be considered average volumes of the cavities (bubbles) that formed during irradiation and were visible in the TEM micrographs. This averaging ignores the influence of small vacancy clusters on swelling.

The amount of swelling was extrapolated from the bubble size and areal density [79]:

$$
\frac{\Delta v}{v}=\frac{4}{3} \pi r_{c}^{3} N_{v} \quad \text { Eq }(2)
$$

where $N_{v}$ is the void density per unit volume and $r_{c}$ is the radius of the voids. It is important to note that this method of estimating swelling ignores the influence of small vacancy clusters since only bubbles observable in TEM were considered. Using the average bubble diameter ( $3 \mathrm{~nm})$ and the areal densities shown in Figure 3a divided by the assumed film thickness $(100 \mathrm{~nm})$, the amount of swelling in the nanocrystalline regime ranges from $0.0096 \%$ to $0.98 \%$ (Figure 7 ). The average bubble diameter $(2.1 \mathrm{~nm})$ and bubble density $\left(0.112 / \mathrm{nm}^{2}\right)$ for the fine grains resulted in swelling of $0.54 \%$. Nanocrystalline grains with sizes less than $2000 \mathrm{~nm}^{2}$ (regime 1) swelled less than the fine grains, while the larger nanocrystalline and ultra-fin grains (regimes 2 and 3) swelled more, as shown in Figure 7. It should be mentioned, however, that these values are subject to variations in Fresnel imaging which we tried to minimize by careful observation of bubble appearance and intensity during under-focusing the beam in the bright field mode. These results, however, suggest that swelling rate can be tuned in nanocrystalline materials and in some cases, the swelling rate in the nanocrystalline materials can exceed that in the fine grained 
materials. Better understanding of grain boundary effects on defect annihilation and densities is crucial for swelling control.

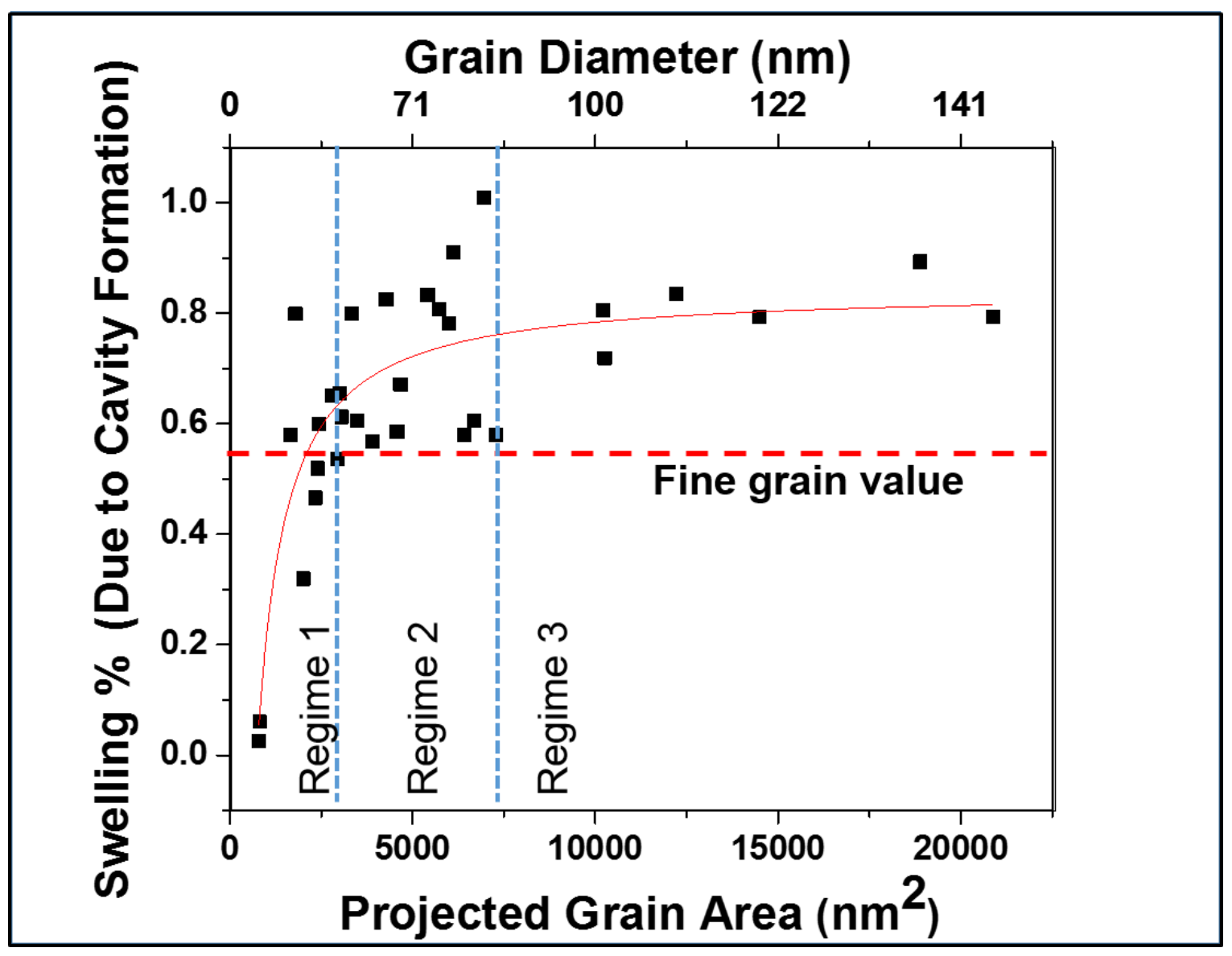

Figure 7: Swelling \% vs grain size (projected grain area) for the three different regimes using the average bubble diameter and the bubble density values (in Figure 3). The dashed line demonstrates the swelling $\%$ found in the fine grains.

\section{Summary and Conclusions}

The performance of nanocrystalline iron in severe irradiation environments was studied using in-situ heating combined with irradiation with low energy helium in a TEM. Estimates of swelling based on bubble formation during the $10 \mathrm{keV}$ helium irradiation at $700 \mathrm{~K}$ were compared to fine grained iron for three grain size regimes. Grains with projected areas less than 
$400 \mathrm{~nm}^{2}$ demonstrated no bubble formation in the grain matrix. Grains with sizes below 3000

$\mathrm{nm}^{2}$ had a trend of bubble density increasing with grain size. Grains with sizes over $7500 \mathrm{~nm}^{2}$ exhibited a slow increasing trend with values approaching saturation near the ultrafine regimes. However, grains in the range between 3000 and $7500 \mathrm{~nm}^{2}$ showed scattering behavior for bubble density, and resultant swelling. In this scattered region, examples of denuded and non-denuded LAGBs, HAGBs and $\sum 3$ boundaries are found, which suggests no dependence of denuded zone formation on grain boundary misorientation angle. Also, channeling cannot be a contributing factor in the scattered distribution because grains with similar orientation and sizes were shown to have very different bubble densities.

Although nanocrystalline grains had lower bubble densities than fine grains, swelling in the nanocrystalline regime was lower than that of the fine grains only for grain sizes below 2000 $\mathrm{nm}^{2}$, due to lower average bubble size in the fine grains.

The results demonstrate the possibility of tuning bubble formation and density in irradiated nanocrystalline iron through grain size refinement. Grains with sizes less than 2000 $\mathrm{nm}^{2}$ (approximately $45 \mathrm{~nm}$ grain diameter) demonstrated lower bubble density and swelling values than ultrafine and fine grains. These fundamental understanding of the role of grain size in a nanocrystalline material in this work enables a science-based development path toward radiation tolerant nanomaterials.

\section{Acknowledgment}

MLT, OEA, ACL, and JEN gratefully acknowledge funding from the United States Department of Energy Basic Energy Sciences (DOE/BES) under the Early Career program through contract DE-SC0008274. This work was performed, in part, at the Center for Integrated Nanotechnologies, an Office of Science User Facility operated for the U.S. Department of 
Energy (DOE) Office of Science by Los Alamos National Laboratory (Contract DE-AC5206NA25396) and Sandia National Laboratories (Contract DE-AC04-94AL85000).Sandia National Laboratories is a multi-program laboratory managed and operated by Sandia Corporation, a wholly owned subsidiary of Lockheed Martin Corporation, for the U.S. Department of Energy's National Nuclear Security Administration under contract DE-AC0494AL85000.

\section{References}

[1] Hemminger J, Fleming G, Ratner M. Directing Matter and Energy: Five Challenges for Science and the Imagination. Department of Energy's Office of Science (http://science energy gov/ /media/bes/pdf/reports/files/gc_rpt pdf) 2007.

[2] Beck CE, Roberts SG, Edmondson PD, Armstrong DE. Effect of alloy composition \& helium ionirradiation on the mechanical properties of tungsten, tungsten-tantalum \& tungsten-rhenium for fusion power applications. MRS Proceedings: Cambridge Univ Press; 2013. p. 99-104.

[3] Zinkle SJ, Busby JT. Structural materials for fission \& fusion energy. Materials Today 2009;12:12-9.

[4] Byun T, Farrell K. Plastic instability in polycrystalline metals after low temperature irradiation. Acta Materialia 2004;52:1597-608.

[5] Zinkle SJ, Was G. Materials challenges in nuclear energy. Acta Materialia 2013;61:735-58.

[6] Lucas $\mathrm{G}$. The evolution of mechanical property change in irradiated austenitic stainless steels. Journal of Nuclear Materials 1993;206:287-305.

[7] Murty K, Charit I. Structural materials for Gen-IV nuclear reactors: challenges and opportunities. Journal of Nuclear Materials 2008;383:189-95.

[8] Kajita S, Sakaguchi W, Ohno N, Yoshida N, Saeki T. Formation process of tungsten nanostructure by the exposure to helium plasma under fusion relevant plasma conditions. Nuclear Fusion 2009;49:095005.

[9] Minyou Y. Effects of low energy and high flux helium/hydrogen plasma irradiation on tungsten as plasma facing material. Plasma Science and Technology 2005;7:2828.

[10] El-Atwani O, Efe M, Heim B, Allain JP. Surface damage in ultrafine and multimodal grained tungsten materials induced by low energy helium irradiation. Journal of Nuclear Materials 2013;434:170-7.

[11] Philipps V. Tungsten as material for plasma-facing components in fusion devices. Journal of Nuclear Materials 2011;415:S2-S9.

[12] Wang Y, Chen M, Zhou F, Ma E. High tensile ductility in a nanostructured metal. Nature 2002;419:912-5.

[13] Wei Q, Zhang H, Schuster B, Ramesh K, Valiev R, Kecskes L, et al. Microstructure and mechanical properties of super-strong nanocrystalline tungsten processed by high-pressure torsion. Acta materialia 2006;54:4079-89.

[14] Kurishita H, Arakawa H, Matsuo S, Sakamoto T, Kobayashi S, Nakai K, et al. Development of nanostructured tungsten based materials resistant to recrystallization and/or radiation induced embrittlement. Materials Transactions 2013;54:456-65.

[15] Shen TD, Feng S, Tang M, Valdez JA, Wang Y, Sickafus KE. Enhanced radiation tolerance in nanocrystalline MgGa 20 4. Applied physics letters 2007;90:263115--3. 
[16] Beyerlein I, Caro A, Demkowicz M, Mara N, Misra A, Uberuaga B. Radiation damage tolerant nanomaterials. Materials today 2013;16:443-9.

[17] El-Atwani O, Hinks J, Greaves G, Gonderman S, Qiu T, Efe M, et al. In-situ TEM observation of the response of ultrafine-and nanocrystalline-grained tungsten to extreme irradiation environments.

Scientific reports 2014;4.

[18] O. El-Atwani AS, TJ. Novakowski, K. Hattar, M. Efe, , S.S Harilal, , A. Hassanein. In-situ TEM/heavy ion irradiation on ultrafine-and nanocrystalline-grained tungsten: effect of $3 \mathrm{MeV} \mathrm{Si}, \mathrm{Cu}$ and $\mathrm{W}$ ions

Materials Characterization, accepted 2014.

[19] Demkowicz M, Hoagland R, Hirth J. Interface structure and radiation damage resistance in $\mathrm{Cu}-\mathrm{Nb}$ multilayer nanocomposites. Physical review letters 2008;100:136102.

[20] Singh BN, Foreman A. Calculated grain size-dependent vacancy supersaturation and its effect on void formation. Philosophical magazine 1974;29:847-58.

[21] Samaras M, Derlet P, Van Swygenhoven + H, Victoria M. Radiation damage near grain boundaries. Philosophical magazine 2003;83:3599-607.

[22] Ackland G. Controlling radiation damage. Science 2010;327:1587-8.

[23] El-Atwani O, Gonderman S, Efe M, De Temmerman G, Morgan T, Bystrov K, et al. Ultrafine tungsten as a plasma-facing component in fusion devices: effect of high flux, high fluence low energy helium irradiation. Nuclear Fusion 2014;54:083013.

[24] Bai X-M, Vernon LJ, Hoagland RG, Voter AF, Nastasi M, Uberuaga BP. Role of atomic structure on grain boundary-defect interactions in Cu. Physical Review B 2012;85:214103.

[25] Bai X-M, Voter AF, Hoagland RG, Nastasi M, Uberuaga BP. Efficient annealing of radiation damage near grain boundaries via interstitial emission. Science 2010;327:1631-4.

[26] Han W, Demkowicz M, Fu E, Wang Y, Misra A. Effect of grain boundary character on sink efficiency. Acta materialia 2012;60:6341-51.

[27] Yu K, Liu Y, Sun C, Wang H, Shao L, Fu E, et al. Radiation damage in helium ion irradiated nanocrystalline Fe. Journal of Nuclear Materials 2012;425:140-6.

[28] Wurster S, Pippan R. Nanostructured metals under irradiation. Scripta Materialia 2009;60:1083-7.

[29] Nita N, Schaeublin R, Victoria M. Impact of irradiation on the microstructure of nanocrystalline materials. Journal of Nuclear Materials 2004;329:953-7.

[30] Chimi Y, Iwase A, Ishikawa N, Kobiyama M, Inami T, Okuda S. Accumulation and recovery of defects in ion-irradiated nanocrystalline gold. Journal of Nuclear Materials 2001;297:355-7.

[31] Rose M, Balogh AG, Hahn H. Instability of irradiation induced defects in nanostructured materials. Nucl Instrum Methods Phys Res, Sect B 1997;127:119-22.

[32] Shen TD, Feng S, Tang M, Valdez JA, Wang Y, Sickafus KE. Enhanced radiation tolerance in nanocrystalline MgGa2O4. Applied physics letters 2007;90:263115-500.

[33] Samaras M, Derlet P, Van Swygenhoven H, Victoria M. Atomic scale modelling of the primary damage state of irradiated fcc and bcc nanocrystalline metals. Journal of nuclear materials 2006;351:4755.

[34] Li J, Yu K, Chen Y, Song M, Wang H, Kirk M, et al. In Situ Study of Defect Migration Kinetics and SelfHealing of Twin Boundaries in Heavy Ion Irradiated Nanotwinned Metals. Nano letters 2015;15:2922-7.

[35] O.El-Atwani KH, J. Hinks, G. Graeme, S. Harilal, A. Hassanein. Helium bubble formation in ultrafine and nanocrystalline tungsten under different extreme conditions. Journal of Nuclear Materials, submitted 2014.

[36] Chen Y, Li J, Yu K, Wang H, Kirk M, Li M, et al. In situ studies on radiation tolerance of nanotwinned Cu. Acta Materialia 2016;111:148-56.

[37] Chen Y, Li N, Bufford D, Li J, Hattar K, Wang H, et al. In situ study of heavy ion irradiation response of immiscible Cu/Fe multilayers. Journal of Nuclear Materials 2016;475:274-9. 
[38] Heintze C, Bergner F, Hernández-Mayoral M, Kögler R, Müller G, Ulbricht A. Irradiation hardening of Fe-9Cr-based alloys and ODS Eurofer: Effect of helium implantation and iron-ion irradiation at $300^{\circ} \mathrm{C}$ including sequence effects. Journal of Nuclear Materials 2016;470:258-67.

[39] Sefta F, Hammond KD, Juslin N, Wirth BD. Tungsten surface evolution by helium bubble nucleation, growth and rupture. Nuclear Fusion 2013;53:073015.

[40] Odette G, Hoelzer D. Irradiation-tolerant nanostructured ferritic alloys: Transforming helium from a liability to an asset. Jom 2010;62:84-92.

[41] El-Atwani O, Gonderman S, Suslov S, Efe M, De Temmerman G, Morgan T, et al. Early stage damage of ultrafine-grained tungsten materials exposed to low energy helium ion irradiation. Fusion Engineering and Design 2015;93:9-14.

[42] Beyerlein I, Demkowicz M, Misra A, Uberuaga B. Defect-Interface Interactions. Progress in Materials Science 2015.

[43] Muntifering B, Blair SJ, Gong C, Dunn A, Dingreville R, Qu J, et al. Cavity Evolution at Grain Boundaries as a Function of Radiation Damage and Thermal Conditions in Nanocrystalline Nickel. Materials Research Letters 2015:1-8.

[44] Deo CS, Okuniewski MA, Srivilliputhur SG, Maloy SA, Baskes MI, James MR, et al. Helium bubble nucleation in bcc iron studied by kinetic Monte Carlo simulations. Journal of nuclear materials 2007;361:141-8.

[45] Borodin V, Vladimirov P. Diffusion coefficients and thermal stability of small helium-vacancy clusters in iron. Journal of nuclear materials 2007;362:161-6.

[46] Scott P. A review of irradiation assisted stress corrosion cracking. Journal of nuclear Materials 1994;211:101-22.

[47] Ullmaier $\mathrm{H}$. The influence of helium on the bulk properties of fusion reactor structural materials. Nuclear Fusion 1984;24:1039.

[48] Fu C-C, Willaime F. Ab initio study of helium in $\alpha-$ Fe: Dissolution, migration, and clustering with vacancies. Physical Review B 2005;72:064117.

[49] Moeck P, Rouvimov S, Rauch E, Veron M, Kirmse H, Häusler I, et al. High spatial resolution semi-automatic crystallite orientation and phase mapping of nanocrystals in transmission electron microscopes. Crystal research and technology 2011;46:589-606.

[50] Vetterick G, Baldwin J, Misra A, Taheri M. Texture evolution in nanocrystalline iron films deposited using biased magnetron sputtering. Journal of Applied Physics 2014;116:233503.

[51] Vetterick GA. Radiation Damage in Nanocrystalline Iron: DREXEL UNIVERSITY; 2014.

[52] Hattar K, Bufford DC, Buller DL. Concurrent in situ ion irradiation transmission electron microscope. Nuclear Instruments and Methods in Physics Research Section B: Beam Interactions with Materials and Atoms 2014;338:56-65.

[53] Ziegler JF, Ziegler MD, Biersack JP. SRIM-The stopping and range of ions in matter (2010). Nuclear Instruments and Methods in Physics Research Section B: Beam Interactions with Materials and Atoms 2010;268:1818-23.

[54] Crocombette J-P, Jourdan T. Cell Molecular Dynamics for Cascades (CMDC): A new tool for cascade simulation. Nuclear Instruments and Methods in Physics Research Section B: Beam Interactions with Materials and Atoms 2015;352:9-13.

[55] Jenkins M. Characterisation of radiation-damage microstructures by TEM. Journal of nuclear materials 1994;216:124-56.

[56] Schneider CA, Rasband WS, Eliceiri KW. NIH Image to ImageJ: 25 years of image analysis. Nat methods 2012;9:671-5.

[57] Lee E, Byun T, Hunn J, Yoo M, Farrell K, Mansur L. On the origin of deformation microstructures in austenitic stainless steel: part I-microstructures. Acta materialia 2001;49:3269-76. 
[58] Arakawa K, Imamura R, Ohota K, Ono K. Evolution of point defect clusters in pure iron under lowenergy He+ irradiation. Journal of Applied Physics 2001;89:4752-7.

[59] Wilson W, Bisson C, Baskes M. Self-trapping of helium in metals. Physical Review B 1981;24:5616.

[60] Singh BN, Leffers T, Green W, Victoria M. Nucleation of helium bubbles on dislocations, dislocation networks and dislocations in grain boundaries during $600 \mathrm{MeV}$ proton irradiation of aluminium. Journal of Nuclear Materials 1984;125:287-97.

[61] Doyle BL. Parameterization of ion channeling half-angles and minimum yields. Nuclear Instruments and Methods in Physics Research Section B: Beam Interactions with Materials and Atoms 2015.

[62] Gomez-Selles JL, Claverie A, Sklenard B, Benistant F, Martin-Bragado I. Atomistic simulation of damage accumulation and amorphization in Ge. Journal of Applied Physics 2015;117:055703.

[63] Lindhard J. Motion of swift charged particles, as influenced by strings of atoms in crystals. Physics Letters 1964;12:126-8.

[64] Onderdelinden D. THE INFLUENCE OF CHANNELING ON Cu SINGLE-CRYSTAL SPUTTERING. Applied Physics Letters 1966;8:189-90.

[65] Kempshall B, Schwarz S, Prenitzer B, Giannuzzi L, Irwin R, Stevie F. Ion channeling effects on the focused ion beam milling of Cu. Journal of Vacuum Science \& Technology B 2001;19:749-54.

[66] Barr CM, Barnard L, Nathaniel JE, Hattar K, Unocic KA, Szlurfarska I, et al. Grain boundary character dependence of radiation-induced segregation in a model $\mathrm{Ni}-\mathrm{Cr}$ alloy. Journal of Materials Research 2015;30:1290-9.

[67] Barr CM, Vetterick GA, Unocic KA, Hattar K, Bai X-M, Taheri ML. Anisotropic radiation-induced segregation in $316 \mathrm{~L}$ austenitic stainless steel with grain boundary character. Acta Materialia 2014;67:145-55.

[68] Zinkle S. Microstructure of ion irradiated ceramic insulators. Nuclear Instruments and Methods in Physics Research Section B: Beam Interactions with Materials and Atoms 1994;91:234-46.

[69] Kesternich W. Helium trapping at dislocations, precipitates and grain boundaries. Radiation effects 1983;78:261-73.

[70] Lane P, Goodhew P. Helium bubble nucleation at grain boundaries. Philosophical Magazine A 1983;48:965-86.

[71] Zinkle S, Kulcinski G, Mansur L. Radiation-enhanced recrystallization in copper alloys. Journal of Nuclear Materials 1986;141:188-92.

[72] Vaidya WV, Ehrlich K. Radiation-induced recrystallization, its cause and consequences in heavy-ion irradiated 20\% cold-drawn steels of type 1.4970. Journal of Nuclear Materials 1983;113:149-62.

[73] Delafond J, Jaouen C, Riviere J, Fayoux C. Ion-beam-induced crystallization of $\mathrm{Ni} 50 \mathrm{Al} 50$ amorphous films. Materials Science and Engineering 1985;69:117-21.

[74] Das G, Mitchell T. Recrystallization induced by electron irradiation of deformed nickel. Scripta Metallurgica 1974;8:1135-40.

[75] Haessner F, Holzer HP. The effect of fast neutron irradiation on the recrystallization of cold-rolled (110)[112] oriented copper crystals. Acta Metallurgica 1974;22:695-708.

[76] Caspers L, Fastenau R, Van Veen A, Van Heugten W. Mutation of vacancies to divacancies by helium trapping in molybdenum effect on the onset of percolation. physica status solidi (a) 1978;46:541-6.

[77] Sizmann R. The effect of radiation upon diffusion in metals. Journal of Nuclear Materials 1978;69:386-412.

[78] Mansur L, Coghlan W. Mechanisms of helium interaction with radiation effects in metals and alloys: A review. Journal of Nuclear Materials 1983;119:1-25.

[79] Mansur L. Theory and experimental background on dimensional changes in irradiated alloys. Journal of Nuclear Materials 1994;216:97-123. 